\title{
Mechanical Design and Position Control of a Modular Mechatronic Device (MechaCell)*
}

\author{
Stefan Ristevski and Melih Çakmakcı, Member, IEEE
}

\begin{abstract}
Manufacturing techniques have advanced exponentially in recent years, providing means for production of smaller and more powerful electronics, which makes it compelling to design small and more powerful robots. Our work focuses on a mechanical design and position control of a modular mechatronic device called MechaCell.

Mechacells are designed as modular semi-autonomous devices which can be used alone or part of a pack. In this paper our main focus is on the mechanical design of the Mechacell, especially the locomotion system which uses forces produced by a rotating unbalance that moves in a spherical domain for steering of the Mechacell. As part of the supervisory algorithm an overhead HD camera is used for position tracking of the Mechacell; the data is then sent to the Mechacells through a wireless connection. A proportional integral derivative controller is used as a base controller; then a friction compensation algorithm is added, based on the mathematical model of the Mechacell's locomotion system. Steering and locomotion controller of the Mechacell is validated using a complex motion profile in the developed testbed.
\end{abstract}

\section{INTRODUCTION}

Popularity of micro systems has been increasing steadily over the recent years due to advances in manufacturing of such small scale systems. Micro (and nano) scale components are usually designed after a long, complicated and expensive process. The tasks are performed by highly trained personnel and the devices are manufactured in extremely specialized facilities with strict external requirements (temperature, humidity, vibration, etc.). Potential application of modular design and development techniques at micro scale introduces many benefits. It will increase the focus of researchers on building one (or fewer different types of) dependable micro module(s) rather than tackling each design problem separately. The development and research effort can then be concentrated on solving common problems. As a result, a mighty and dependable micro module can be mass-produced at an affordable price, which can be used in many different applications. Using one type (or few types) of micro mechatronic components will increase the number of skilled developers who are familiar with the component's mechanical and software structure.

*Research supported by European Commission through Seventh Framework Program for Research and Technological Development.

Stefan Ristevski (corresponding author) is with the Mechanical Engineering Department of Bilkent University, Ankara, 06800 TURKEY (phone: +90-312-290-3066; e-mail: stefan.ristevski@ bilkent.edu.tr).

Melih Cakmakci is with the Mechanical Engineering Department of Bilkent University, Ankara, 06800 TURKEY (phone: +90-312-290-3427; e-mail: melihc@bilkent.edu.tr).
Conceptually, the proposed micromechatronic device will have an outer shell with multiple contact interfaces that handle mechanical and electrical interaction among devices in a pack. It would include a micro-power plant (and storage) unit, microsensors and actuators as well as a microcomputer, all of which are connected to a cell bus handling both power and communication among the cell components. A group of these Mechacells (Mechacell Pack) can perform tasks under different conditions, sharing mechanical, electrical and computing resources. Some of the technologies that will make the mass produced micro-mechatronic cells feasible are currently under development or available at a very large cost. A good example of this is the availability of micropower plants.

The idea of building components from highly dependable and affordable modular components is welldefined, studied and successfully implemented concept. Its implications have been considered by many researchers [1], [2]. Most mechatronic systems in the macro scale are built from off the shelf components i.e. sensors, actuators and controllers. With the increasing availability of electronics at a much lower cost, actuators/sensors themselves have computing power, in some cases network communication capabilities as well; this makes them stand-alone mechatronic modules [3], [4], [5], [6]. Successful implementation of building robots from common modules (robotic stem cells) is discussed in [7]. In [8] a review of the current state of the art, challenges and opportunities for these types of robotic systems ia also presented. The work presented here differs from the work mentioned, such that it focuses on building a mechatronic module (with onboard storage) which will be used as the building block for complex mechatronic systems. The objective of the research presented here is to design modular mechatronic semi-autonomous device-the Mechacell, whose pack will be able to position and orient an object in a plane by applying forces on its perimeter. The distributed and modular position control system design will make it possible to use relatively simple and less capable mechatronic devices cooperatively. Distributed position control is a good example to illustrate the cooperative work of these mechatronic devices. In the literature, there are various approaches to the positioning problem. In [11] and [12], behavior based approaches are used for non articulated cooperative manipulation of objects. In [13]-[15] an object is surrounded by mobile robots using a caging approach, which constrains inter robot spacing and makes escape impossible 
for the object. It is assumed that object moves when the robots move.

The rest of the paper is organized as follows: Section 2 presents an overview of the Mechacell device and its design principles. Testbed, position tracking system and a mathematical model are included as well. In Section 3, our control design approach of the system is explained. Section 4 presents experimental results and discussions. In addition, controller performance metrics are given. Finally conclusion and future work are discussed in Section 5.

\section{Design Of The Mechacell And Testbed}

In this section, primary design stages of the MechaCell are presented. Our objective is to design a simple, easily manufactured and assembled modular device. Our space requirement is to fit everything (actuators, processing - units, communication hardware etc.) into packaging constrained to $(5 \times 5 \times 5) \mathrm{cm}^{3}$, with the ability to miniaturize the whole design in later stages. Additive manufacturing is a simple, fast and reliable manufacturing process, hence all mechanical components of the Mechacell are manufactured with a 3Dprinter.

\section{A. Mechanical Design and Locomotion}

In Figures (1) and (2), all parts of our device are presented. Components are named and numbered accordingly. Excluding the actuators (two vibrational motors and one servo motor) there are ten mechanical parts that are manufactured with additive manufacturing process. Some of the mechanical parts presented in Figure (1) and (2) are manufactured separately to make manufacturing and assembly easier. Figure (3a) and Figure (3b) present CAD model drawn in CAD software and the actual (manufactured) Mechacell respectively.

Locomotion mechanism consists of two separate and mutually independent sections: translational section and rotational section. Translational section translates the Mechacell in the $x y$-plane, refer to Figure (4), and rotational section rotates the Mechacell about its own axis.

A novel approach has been taken in the design of the locomotion mechanism of this module. Vibrational forces produced by a rotating unbalance are being used. This locomotion mechanism differs from the locomotion mechanism of bristle bots [20] in two ways: 1-it does not use any bristles; 2-in bristle bot, locomotion mechanism only depends on the vertical component of the vibrational force, where locomotion mechanism here depends on both, the vertical and horizontal components of the vibrational force.

\section{B. Main and Accessory Electronics}

Main processing unit is an Arduino ProMini board, [17]. Half $\mathrm{H}$ bridges are used to drive the vibrational motors in both directions (clock-wise and counter-clock-wise). Speed of the vibrational motor is controlled by the Arduino's PWM signals [18]. Integrated Bluetooth module establishes communication link between the PC and the Mechacell.

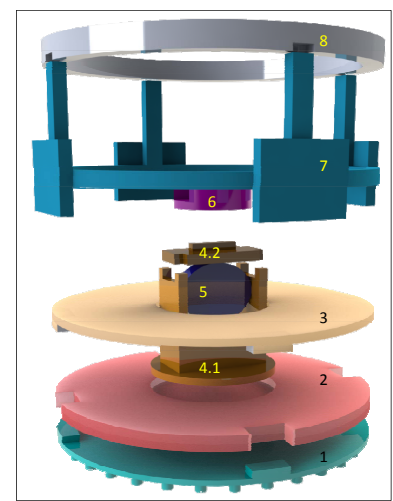

1. Locomotion mechanism part -1 2. Locomotion mechanism part - 2

3. Locomotion mechanism part -3

4. Locomotion mechanism part -4 (translational

vibrational motor fixture)

5. Translational vibrational motor

6. Locomotion mechanism part -5 (coupling)

7. Circumference fence

8. Circumference ring

Fig. 1. Assembly steps part 1

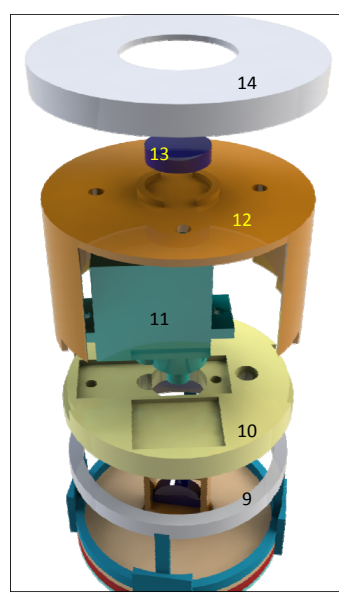

9. Circumference ring 10.Servo motor fixture 11.Servo motor

12.Top cover (electronics cover)

13. Rotational vibrational motor 14.Rotational vibrational motor cor

Fig. 2. Assembly steps part 2

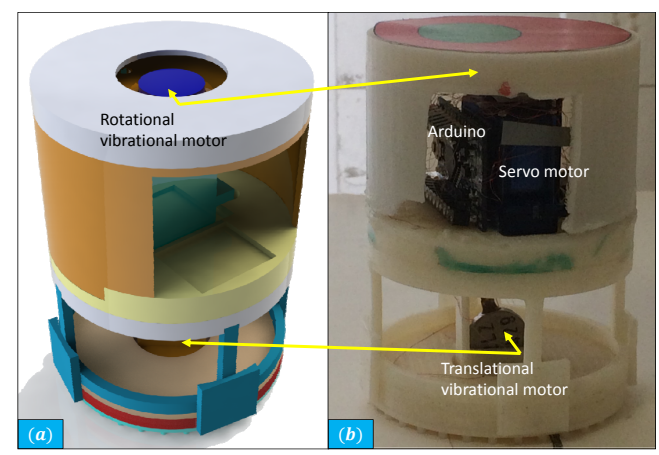

Fig. 3. (a) Cad model of the Mechacell (b) Mechacell prototype 


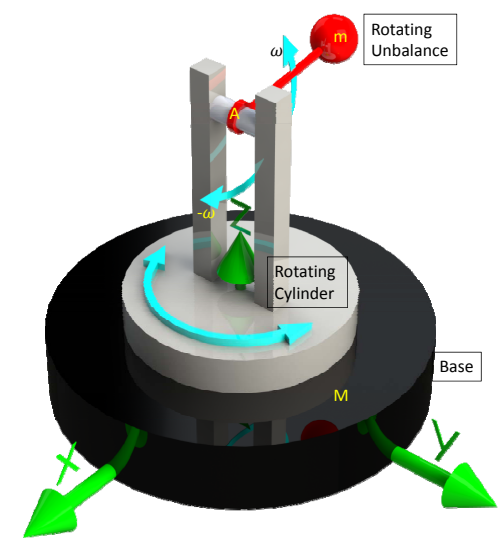

Fig. 4. Rotating unbalance with mass $m$ attached to a block of mass $M$ at point $A$

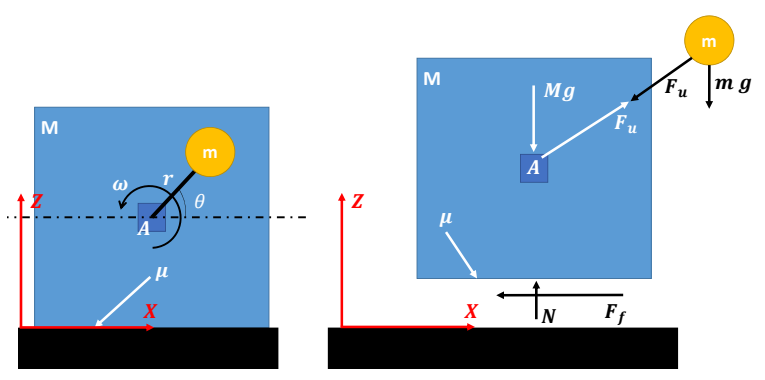

Fig. 5. Planar representation of the rotating unbalance shown in Figure (4)-left; free body diagram-right

\section{Mathematical Model}

Figure (4) presents a simplified version of the locomotion system, rotating unbalance of mass $m$ attached to a cylinder with mass $M$ at point $A$, rotating with constant angular speed $\omega$ and constrained to a spherical motion for steering. Figure (5), left presents planar view of the rotating unbalance shown in Figure (4), right presents a free body diagram according to the figure in the left. Friction force acting on the system is modeled by using Coloumb's friction model with static and dynamic constants. Magnitude of the force produced by the rotating unbalance depends on the angular speed, $\omega$, and is presented in (1) with its projections on $x$ and $z$ axis w.r.t. Figure (4), and in (2) friction force magnitude is presented.

$$
\begin{gathered}
F_{u}(\omega)=\omega^{2} m r \\
F_{u x}(\omega)=F_{u} \cos (\omega t)=\omega^{2} m r \cos (\omega t) \\
F_{u z}(\omega)=F_{u} \sin (\omega t)=\omega^{2} m r \sin (\omega t) \\
F_{f}=N \mu_{s} \text { (not in motion) } \\
F_{f}=N \mu_{k} \text { (in motion) } \\
N(\omega)=M g+\omega^{2} m r \sin (\omega t)
\end{gathered}
$$

In Figure (6), comparison between the mathematical model and experimental data from the real model is shown. As stated above, angular speed depends on the PWM signal, hence in Figure (6), the range of the signal is swept for model validation.
TABLE I

PARAMETERS USED IN THE MATHEMATICAL MODEL

\begin{tabular}{|c||c||c|}
\hline Mass of the Mechacell & $M$ & $0.049[\mathrm{~kg}]$ \\
\hline Mass of the rotating unbalance & $m$ & $0.006[\mathrm{~kg}]$ \\
\hline Radius of the rotating unbalance & $r$ & $0.004[\mathrm{~m}]$ \\
\hline Angular speed of the unbalance & $\omega$ & swept $[\mathrm{rad} / \mathrm{s}]$ \\
\hline Coefficient of static friction & $\mu_{s}$ & 0.2 \\
\hline Coefficient of kinetic friction & $\mu_{k}$ & 0.15 \\
\hline Force produced by the unbalance & $F_{u}$ & calculated $[N]$ \\
\hline Friction force & $F_{f}$ & calculated $[N]$ \\
\hline Normal force & $N$ & calculated $[N]$ \\
\hline Gravitational constant & $g$ & $9.81\left[\mathrm{~m} / \mathrm{s}^{2}\right]$ \\
\hline
\end{tabular}
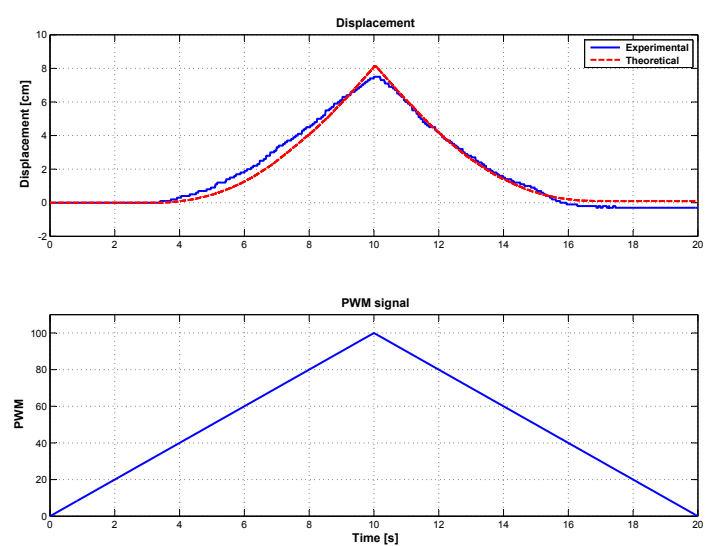

Fig. 6. Comparison of the mathematical model to real one

In Table (I) all parameters with numerical values (if applicable) used in the simulation of the mathematical model are presented.

\section{Testbed and Position Tracking}

An overhead HD camera is used to track the position of each Mechacell. Information about own position, positions about the rest of the active Mechacells in the system and position of the workpiece (desired position included) is sent to each Mechacell. Real time video from the overhead camera is processed with Matlab/Simulink Image Acquisition and Computer Vision toolboxes. Using color based object tracking algorithm, positions and orientations of all Mechacells, and a workpiece are calculated. Figure (10) presents color pattern that is printed on the top of each Mechacell for position/orientation tracking and differentiation between Mechacells (each mechacell is coded differently). The algorithm finds the centroids of same-colored connected regions. Using centroids of the colors that a Mechacell is coded with, Mechacells position/orientation can be obtained. Centroid of the background color is the position of the Mechacell, and orientation of the vector starting at the centroid of the background color and ending at the centroid of the foreground color is the orientation of the Mehcacell, i.e. orientation of vector $\mathbf{R}_{\mathbf{B} / \mathbf{A}}$ in Figure (10). Vectors $\mathbf{R}_{\mathbf{A}}$ and $\mathbf{R}_{\mathrm{B}}$ present centroid position vectors of both background and foreground colors respectively. Vector $\mathbf{R}_{\mathbf{B} / \mathbf{A}}$ is given in (4). 

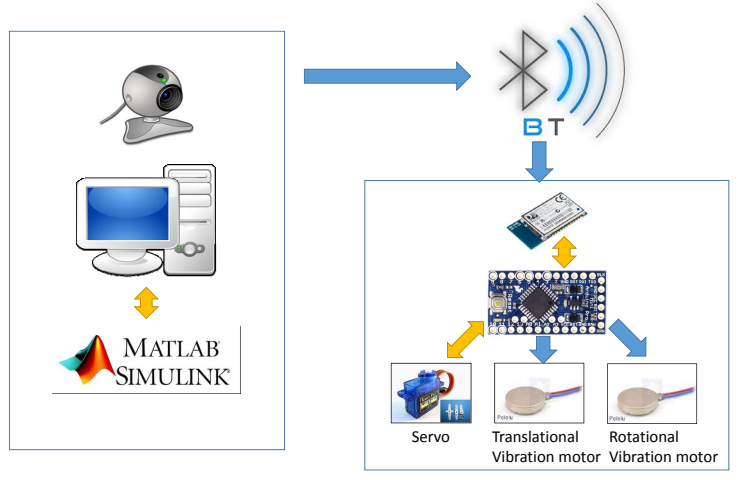

Fig. 7. Schematics of PC (Matlab/Simulink) communication with Mechacell through Bluetooth

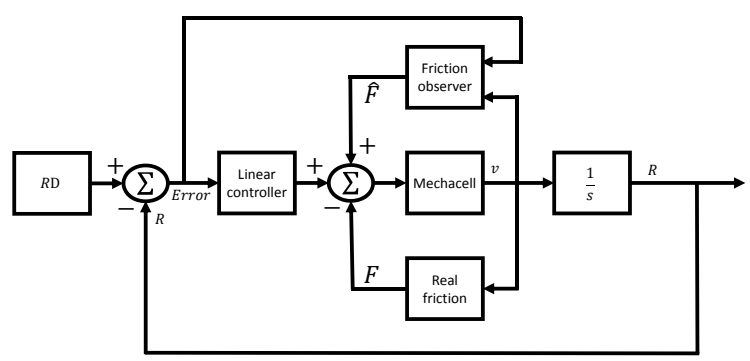

Fig. 8. Block diagram of a control scheme with friction compensation

$$
\mathbf{R}_{\mathrm{B} / \mathrm{A}}=\mathbf{R}_{\mathrm{B}}-\mathbf{R}_{\mathrm{A}}
$$

Using $x$ and $y$ component of vector $\mathbf{R}_{\mathbf{B} / \mathbf{A}}$, orientation of the Mechacell, $\theta$ is calculated in (5).

$$
\theta=\operatorname{atan} 2\left(R_{B / A}(y), R_{B / A}(x)\right)
$$

\section{Translational and SteERING Control ALGORITHM}

The Mechacell is equipped with two independent controllers, a t-controller and an $\mathrm{r}$-controller, responsible for translation and rotation respectively, Figure (9). Both tcontroller and r-controller consist of two sub-controllers. Sub-controller 1 of the t-controller is a PID controller (tPID) with friction compensation algorithm [19] controlling translational actuating force's magnitude, Figure (8). Subcontroller 2 of the $t$-controller is an algorithm that calculates the angle of the servo motor and the direction of rotation of the vibrational motor, controlling translational actuating force's direction.

Sub - controller 1 of the $r$ - controller is a PID controller ( $r$ PID) controlling the rotational speed of the vibrational motor (rotational actuating moment-magnitude), and sub-controller 2 is an algorithm that controls the direction of rotation of the vibrational motor (rotational actuating moment-direction).

Error signals $e_{t}$ and $e_{r}$ that are inputs to t-PID and r-PID controllers are calculated in (6) and (7) respectively.

$$
\begin{aligned}
\mathbf{R}_{\mathrm{MD}} & =\mathbf{R}_{\mathbf{D}}-\mathbf{R}_{\mathbf{M}} \\
e_{t} & =\left|\mathbf{R}_{\mathrm{MD}}\right|
\end{aligned}
$$

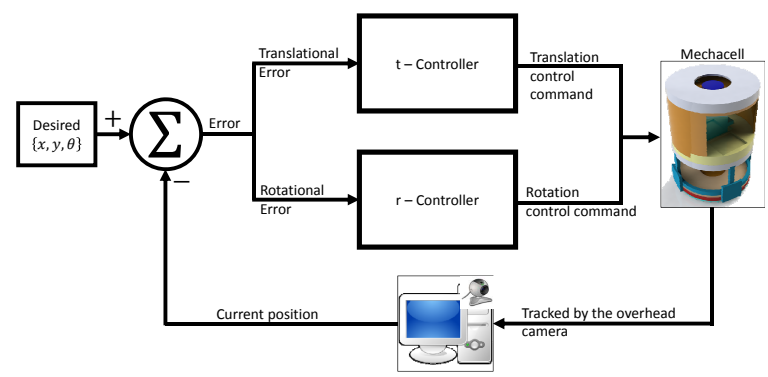

Fig. 9. Closed loop control with Translational (t-controller) and Rotational (r-controller)

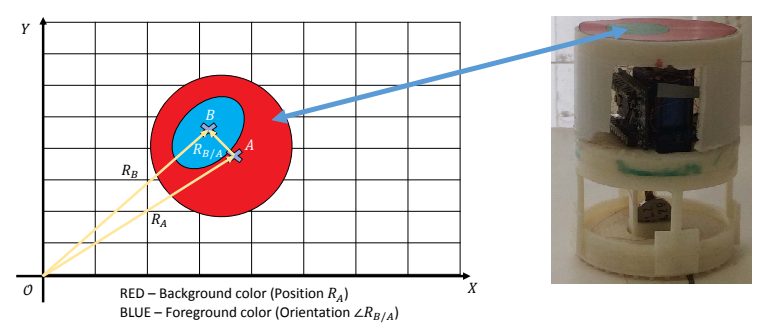

Fig. 10. Mechacell color coding for position and orientation tracking

$$
e_{r}=\left|\theta_{D}-\theta\right|
$$

Where $\theta_{D}$ is the desired orientation, and $\theta$ is current orientation of the Mechacell given in (5).

Figure (11) shows the top view of a Mechacell. Also in this figure, Mechacell's orientation, desired position in the $x y$-plane and orientation of the servo motor are shown too.

Sub-controller 2 of the t-controller, calculates the Servo motors orientation according to (8).

$$
\alpha_{\text {servo }}=\alpha_{D}+\left(\alpha_{M}-90^{\circ}\right)
$$

Where, $\alpha_{D} \equiv \angle \mathbf{R}_{\mathrm{MD}}, \alpha_{\text {servo }} \equiv \angle A M E, \alpha_{M} \equiv$ $\angle B M C$, Figure (11). Direction of the vibrational motor is reversed if vector $\mathbf{R}_{\mathrm{MD}}$ does not intersect arc $A C F$ in Figure (11). If direction of the vibrational motor is reversed, servo motor's angle is recalculated by using negative of the vector $\mathbf{R}_{\mathrm{MD}}$.

Sub-controller 2 of the r-controller reverses the direction of rotation of the vibrational motor when condition in (9) is not satisfied. 


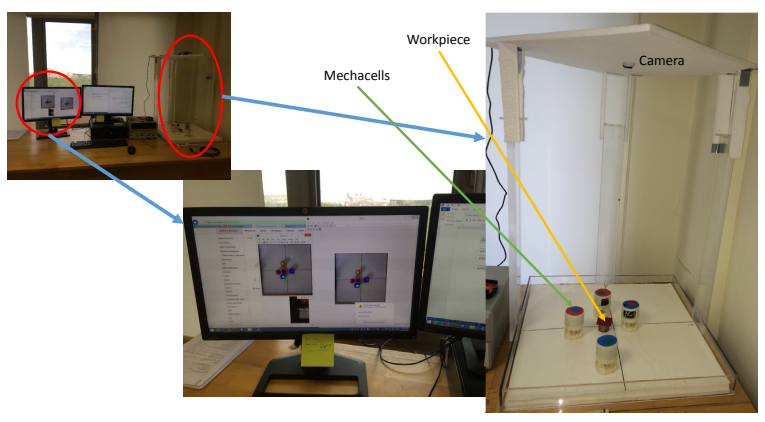

Fig. 12. Experimental Setup: PC with running tracking algorithm on the left; overhead camera and testbed platform on the right

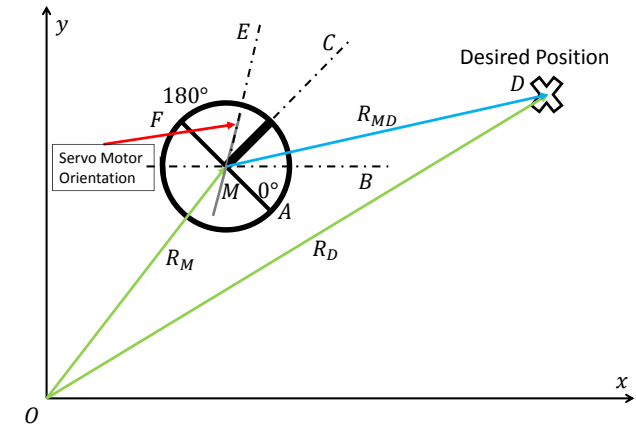

Fig. 11. Servo motor orientation calculation algorithm

\section{EXPERIMENTS AND RESUlts}

Experimental setup consists of a platform with overhead HD-camera, a PC with Matlab/Simulink and Bluetooth, and Mechacells, Figure (12). Real time video from the camera is used by the color tracking algorithm running in Simulink, this algorithm calculates position and orientation of the Mechacell, and sends the data through Bluetooth to the Mechacell.

Figure (13), shows positioning of a Mechacell from 0 to $1 \mathrm{~cm}$ by using different t-PID controller configurations (excluding friction compensation algorithm). T-PID controller configurations are varied such that: left to right proportional control changes from 60 to 90 with increments of 10 , and integral control changes top to bottom from 0.05 to 0.15 with increments of 0.05 , derivative control is fixed at 0.1 .

Figure (14) is an enlarged view of subplot 4 of Figure (13). Response 4 in Figure (13) is fast and has no overshoot, hence is considered as best response compared to the rest of the responses in Figure(13). Figure(14) is enlarged view of response 4 (blue solid line), in the same figure a response with added friction compensation algorithm is presented (green dashed line). It can noticed that friction compensation algorithm increases the speed of the response.

Using the best t-PID controller configuration with friction compensation algorithm in the translational sub-controller, a more complicated experiment is performed, Figure (16). This figure presents a Mechacell tracking a $B$-shaped path. The $B$-shaped path is formed from a total of 36 points connected with linear segments. Combination of straight lines with
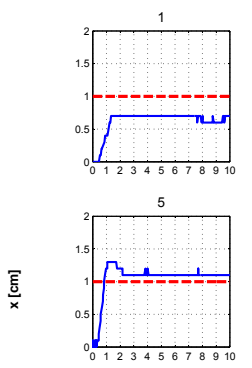

9
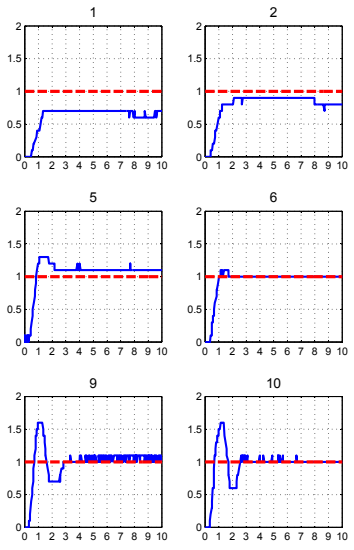

10
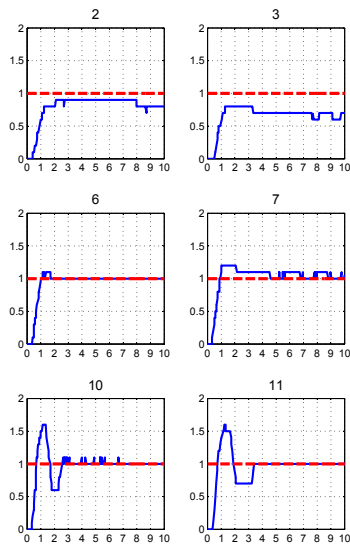

11

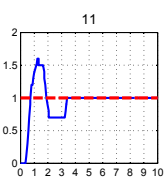

$\mathrm{t}[\mathrm{s}]$

Fig. 13. Response of different t-PID settings

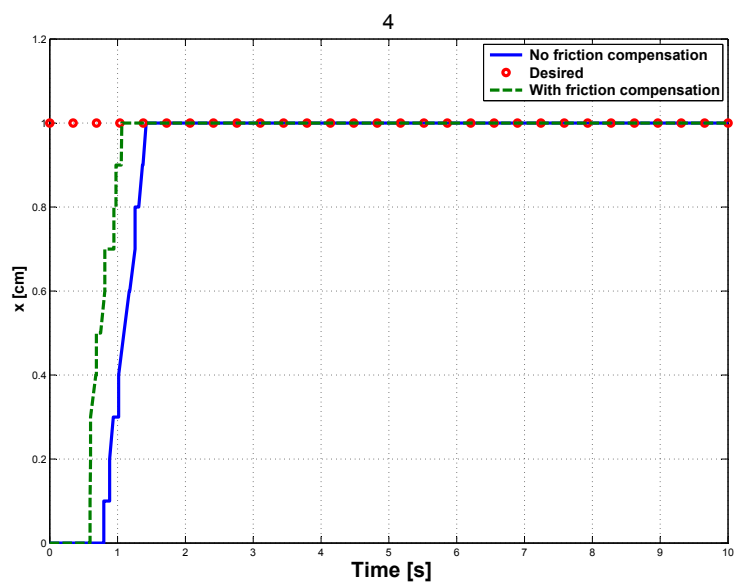

Fig. 14. Step response: t-PID with friction compensation algorithm (green dashed line); t-PID without friction compensation algorithm (blue solid line)

curved lines in addition to sharp turns makes this shape a complex path to be tracked and a good candidate to validate our steering and control design. According to the control scheme is Figure (9), desired position is updated every $1[s]$, while keeping the desired orientation of the Mechacell constant, $\theta_{D}=45^{\circ}$.

Comparing current position to desired position at every second, deviations in $x$ and $y$ directions are calculated, $x E r r$ and $y E r r$ respectively. Shortest distance from the current position to the contour is defined as contour error, $\epsilon$, Figure (15). Using RMS averaging of the parameters above, performance of the controller is calculated, $[x E r r, y E r r, \epsilon]=[0.4685,0.3338,0.1237][\mathrm{cm}]$. All errors are in sub-centimeter scale which is the performance limits of our project requirement.

\section{CONCLUSiON}

In this paper design of a modular mechatronic device, the Mechacell is presented. The design offers a different approach in the design of the locomotion system, it uses forces produced by a rotating unbalance and is different from the locomotion system in bristle bots. This locomotion 


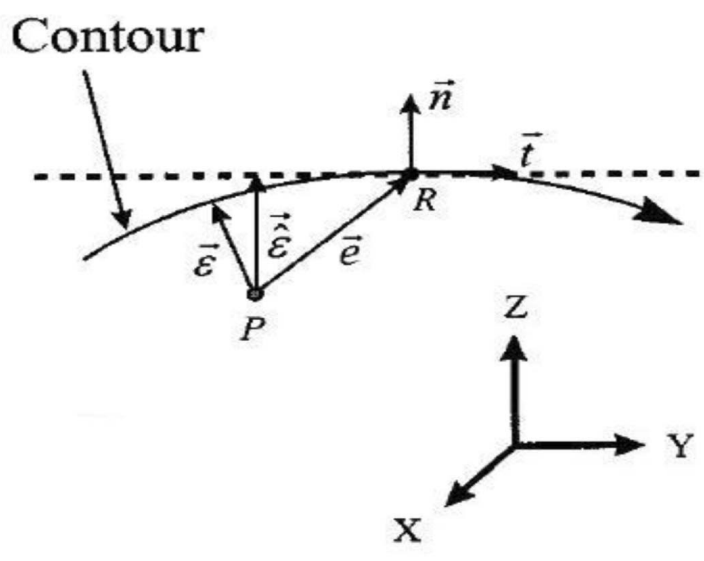

Fig. 15. Contour and tracking error

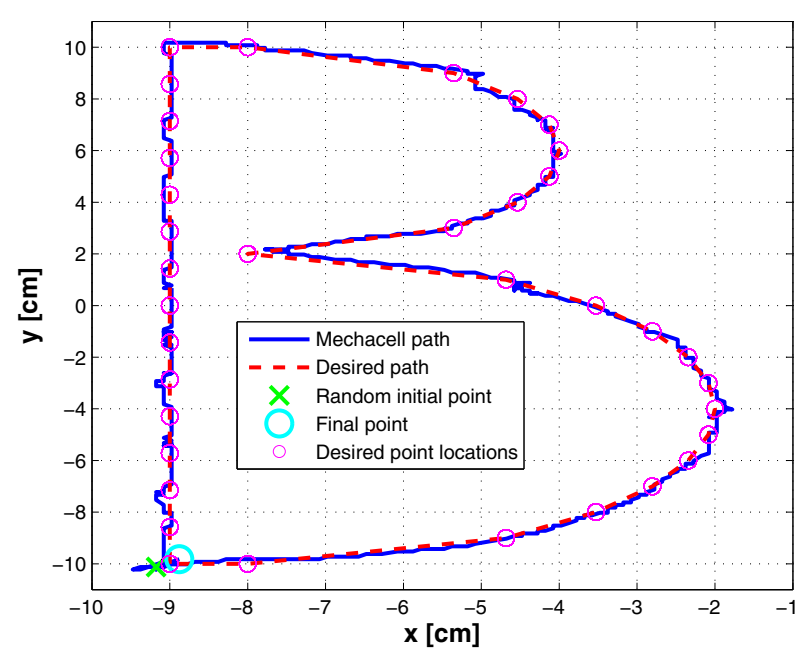

Fig. 16. $B$ - shaped path tracked by a Mechacell

system uses common off the shelf shaftless vibrational motors and a servo motor and is easily implementable. All mobile parts of the locomotion systems are concealed inside an outer cylindrical body of the Mechacell which is advantageous if size of the device is to be considerably reduced. In addition to the mechanical design, this paper offers a controller design for locomotion and steering with friction compensation algorithm.

Locomotion and steering experiments were carried out and results were presented. The device was able to position itself as desired using the steering system and algorithm, also the device was able to follow a more complex path (combination of linear and non-linear line segments with sharp turns), a Bshaped path. Using different error parameters, performance of the controller is calculated. The resulting device has subcentimeter range positioning performance which satisfies our project requirement.

Future work will include manufacturing more Mechacells and investigating swarm behavior, also future work will include reducing the size of the Mechacell, design of a miniMechacell.

\section{REFERENCES}

[1] Ulrich K., and Tung K., 1991, Fundamentals of Product Modularity, Issues in Mechanical Design International, A. Sharon, ed., ASME, New York, pp. 7379.

[2] Salerno M. S., and Dias A. V. ., 2002, Product Design Modularity, Modular Production, Modular Organization: The Evolution Of Modular Concepts, Actes du GERPISA no 33, pp. 6173.

[3] Inc D. N., Dust Networks Website.

[4] Corporation F., Flowserve Website.

[5] Isermann R., and Raab U., 1993, Intelligent actuators: ways to autonomous actuating systems, Automatica (Journal of IFAC), 29(5), pp. 13151331.

[6] Liu J., Chu M., Reich J., and Zhao F., 2003, State-centric programming for sensor-actuator network systems, IEEE Pervasive Computing, 2(4), pp. 5062.

[7] Rubenstein M., Sai Y., Chuong C. M., And Shen W., 2009, Regenerative patterning in Swarm Robots: mutual benefits of research in robotics and stem cell biology, Int. J. Dev. Biol, 53(5-6), pp. 869881.

[8] Mark Y., Shen W., Salemi B., Daniela R., Moll M., Lipson H., And Klavins E., 2007, Modular Self-Reconfigurable Robot SystemsChallenges and Opportunities for the Future, IEEE Robotics \& Automation Magazine, 14(1), pp. 4352.

[9] Bishop, B.E., 2003. On the Use of redundant Manipulator Techniques for Control of Platoons of Cooperating Robotic Vehicles. IEEE Transactions on Systems, Man and Cybernetics, Part A: Systems and Humans, 33(5), pp. 608615

[10] Fossen, T.I., 1994. Guidance and Control of Ocean Vehicles, John Wiley \& Sons, Norway, Chap. 2, pp. 48-55.

[11] Song, P., Kumar, V., 2002. A Potential Field Based Approach to Multirobot Manipulation. IEEE International Conference on Robotics and Automation, pp. 1217-1222.

[12] Kube, C.R., Bonabeau, E., 2000. Cooperative Transport by Ants and Robots, Robotics and Autonomous Systems, 30(1-2), Jan., pp. 85-101.

[13] Sudsang, A., Ponce, J., 2000. A New Approach to Motion Planning for Disc-shaped Robots Manipulating a Polygonal Object in the Plane. IEEE International Conference on Robotics and Automation, pp. 1068 1075 .

[14] Wang, Z., Nakano, E., Takahashi, T., 2003. Solving Function Distribution and Behavior Design Problem for Cooperative Object Handling by Multiple Mobile Robots. IEEE Transactions on Systems, Man and Cybernetics, Part A: Systems and Humans, 33(5), Sep., pp. 537- 549.

[15] Pereira, G.A.S., Campos, M.F.M., Kumar, V., 2004. Decentralized Algorithms for Multi-Robot Manipulation via Caging. The International Journal of Robotics Research, 23(7-8), Aug., pp. $783-795$.

[16] Sugar, T.G., Kumar, V., 2002. Control of cooperating mobile manipulators, IEEE Transactions on Robotics and Automation, 18(1), Feb, pp. 94-103.

[17] http://www.arduino.cc/

[18] F. Vasca and N. Ianneill, "Chapter 2: Pulse width modulation," in Dynamics and control of switched electronic systems Advanced perspectives for modeling, simulation and control of power, Springer, 2012, p. 494.

[19] H. Olsson, K. J. strm, M. Gfvert, C. Canudas De Wit and P. Lischinsky, 1998. "Friction models and friction compensation". Eur. J. Control.

[20] G. Cicconofri and A. DeSimone, 2014. "Motility of a model bristlebot: A theoretical analysis". International Journal of Non-Linear Mechanics 